\title{
Análisis Organizacional y Financiero del uso de Prácticas Sustentables en Empresas Industriales Hidalguenses
}

\author{
Dr. Sergio Demetrio Polo Jiménez, \\ Profesor Investigador del Área Académica de Contaduría de la Universidad \\ Autónoma del Estado de Hidalgo, México \\ P.L.C. Daniela Martín Benitez, \\ Alumna del Noveno Semestre de la Licenciatura en Contaduría de la \\ Universidad Autónoma del Estado de Hidalgo, México \\ Dr. Heriberto Moreno Islas, \\ Mtra. Martha Jiménez Alvarado, \\ Profesor Investigador del Área Académica de Contaduría de la Universidad \\ Autónoma del Estado de Hidalgo, México
}

Doi:10.19044/esj.2020.v16n22p37 ～URL:http://dx.doi.org/10.19044/esj.2020.v16n22p37

\section{Resumen}

El propósito de la presente investigación, se orienta en conocer si las prácticas sustentables (ahorro de energía eléctrica), realizadas en una muestra de 10 empresas industriales pertenecientes a dos municipios del Estado de Hidalgo, México, influyen en sus estructuras financieras. Para alcanzar los objetivos planteados se calculan dos índices, uno relativo al desarrollo de prácticas sustentables (IPS) y otro, relativo al aspecto financiero (IFIN), ambos similares al propuesto por (Céspedes, (2001); Carrillo, (2014); Vázquez et al., (2011). Los resultados de la aplicación del modelo de regresión lineal, muestran una relación estadísticamente significativa de tipo positivo entre el Índice Financiero (IFIN) y las variables relativas al grado de escolaridad del responsable, número de trabajadores, el (IPS). En definitiva, los resultados nos podrían permitir inferir, en que las empresas que implementan prácticas sustentables, impactan sustancialmente sus estructuras financieras.

Palabras clave: Sustentabilidad, índice de sustentabilidad, regresión lineal, prácticas de ahorro de energía 


\title{
Organizational and Financial Analysis of the Use of Sustainable Practices in Industrial Companies from Hidalgo
}

\author{
Dr. Sergio Demetrio Polo Jiménez, \\ Profesor Investigador del Área Académica de Contaduría de la Universidad \\ Autónoma del Estado de Hidalgo, México \\ P.L.C. Daniela Martín Benítez, \\ Alumna del Noveno Semestre de la Licenciatura en Contaduría de la \\ Universidad Autónoma del Estado de Hidalgo, México \\ Dr. Heriberto Moreno Islas, \\ Mtra. Martha Jiménez Alvarado, \\ Profesor Investigador del Área Académica de Contaduría de la Universidad \\ Autónoma del Estado de Hidalgo, México
}

\begin{abstract}
This paper focuses on ascertaining if the sustainable practices (saving of electrical energy), carried out in a sample of 10 industrial companies belonging to two municipalities of the State of Hidalgo, Mexico, have a significant influence on their financial structures. To achieve the proposed objectives, two indices were calculated, one related to the development of sustainable practices (IPS) and the other related to the financial aspect (IFIN). Both were similar to the one proposed by Céspedes (2001), Carrillo (2014), and Vázquez et al. (2011). The results of the application of the linear regression model show a statistically significant positive relationship between the Financial Index (IFIN) and the variables related to the level of education of the person responsible, number of workers, and the IPS. The results show that companies that implement sustainable practices have a substantial impact on their financial structures.
\end{abstract}

Keywords: Sustainability, sustainability index, linear regression, energy saving practices

\section{Introduction}

El cuidado y protección al medio ambiente y los recursos naturales, ha despertado el interés en diversos organismos, como es el caso de la Organización de las Naciones Unidas (ONU), quien ha manifestado, su 
preocupación por dichas cuestiones. En la Conferencia de la ONU, sobre el Desarrollo Sostenible (Río+20, 2012), refiere la necesidad de garantizar la protección del medio ambiente, considerando que la población se ha incrementado de manera gradual y exponencial en los últimos años. Río+20, enfatiza en la construcción de una economía ecológica que promueva aspectos sustentables mediante un desarrollo sostenible. En el mismo sentido, el Decenio Internacional para la Acción (El agua fuente de vida 2005-2015), perteneciente también a la ONU, establece que el consumo de agua y la producción de energía eléctrica, se encuentran intrínsecamente relacionadas, lo cual se respalda con el Informe sobre el Desarrollo de los Recursos Hídricos en el Mundo (2014), que resalta como indispensable, la participación activa de los gobiernos y las unidades del sector privado en la investigación y desarrollo de energías alternativas. Begoña (2010), con la intención de proporcionar una guía del consumo y gestión eficiente de la energía a los trabajadores de diversos sectores de la economía, hace una clara distinción entre los conceptos de consumo y servicio energético. Definiendo el consumo energético como la cantidad de energía que debe ser consumida para producir una unidad de determinado producto; y el servicio energético como un consumo racional y eficiente que busque producir lo requerido sin abusar del uso de la energía y disminuyendo, a su vez, el impacto ambiental negativo mediante emisiones contaminantes.

La revisión de la literatura, muestra que el tema de la Responsabilidad Social Empresarial (RSE), ha derivado del interés del sector empresarial siendo un tema que continua en construcción por lo que, sus prácticas proporcionan bases sobre las directrices para que las empresas asuman comportamientos responsables (Agüero, 2002). Desde hace algunos años se han realizado estudios que permiten dar a conocer el comportamiento socialmente responsable de las empresas. En este sentido, muchas organizaciones colaboran con las empresas para poner en marcha campañas con el fin de verificar la efectiva aplicación de estándares de regulación económica y social, que garanticen la observancia de los derechos humanos, laborales y medio ambientales. Estos requerimientos se insertan en la denominada teoría institucional de la RSE, que vislumbra el grado de aceptación que la sociedad tiene hacia las instituciones. Dentro de la aceptación que las empresas buscan, se encuentra la Responsabilidad Social hacia los Stakeholders (Arreguín, Álvarez \& Sánchez, 2019, p.52) y han tenido como resultado junto con otros factores, que las empresas elaboren instrumentos como códigos de conducta, memorias de RSE y auditorías sociales.

Referente a los impactos medioambientales, aunque a primera vista los ocasionados por las grandes empresas pueden parecer más evidentes y de mayor dimensión que los de una PYME (y, por lo tanto, tengan una mayor 
responsabilidad para con la sociedad), lo cierto es que las pequeñas y medianas empresas también ejercen importantes presiones sobre el medio ambiente, y por lo tanto también tienen que adoptar un compromiso social al respecto. El alto porcentaje de pymes en México, presentan un potencial de ahorro de energía considerable, por lo que la incorporación de medidas que ayuden a mejorar la gestión energética y buenas prácticas ambientales en los centros de trabajo, ayudarían a optimizar de forma notoria la imagen de las empresas de cara a los ciudadanos y a darle un valor añadido a sus organizaciones en un mundo empresarial, cada vez más implicado con la protección del medio ambiente.

En este sentido, el presente trabajo de investigación, se orienta en analizar si las prácticas ahorradoras de energía eléctrica desarrolladas por una muestra de empresas industriales hidalguenses, promueven el ahorro financiero, identificando aquellos factores condicionantes para su realización. Para alcanzar los objetivos propuestos, se estructura el estudio de la siguiente manera: tras la introducción, el trabajo dedica el siguiente apartado a la revisión de la literatura previa; posteriormente se da a conocer la metodología aplicada, describiendo la muestra y definiendo las variables objeto de estudio; en el cuarto apartado se describen los resultados obtenidos y terminamos con la exposición de las principales conclusiones y referencias.

\section{Revisión de la literatura}

La sustentabilidad es un proceso que se orienta en conocer la relación que existe entre el medio ambiente y el uso de los recursos naturales. Concepto que surge en 1987 por la Comisión del Medio Ambiente y Desarrollo para la Organización de Naciones Unidas y presentado en el informe "Nuestro futuro en común", que está centrado en la idea del desarrollo sustentable, que se basa en satisfacer las necesidades de las personas siempre y cuando no se comprometa a las futuras generaciones (Centro del Cambio Global \& la Sustentabilidad, 2020). De esta manera, la sustentabilidad de las organizaciones gira entornos a tres direcciones: la ambiental, la económica y la social, lo cual genera en ellas compromiso, innovación, competitividad e internacionalización, considerando las consecuencias que estas actividades tienen sobre los grupos de interés (Anzures, Briones \& Galarza, 2017; Briseño, Lavín \& García, 2011; De la Cuesta \& Valor, 2003).

Es abundante la literatura que se ha encargado de explicar la relación existente entre las actividades realizadas para la conservación del medio ambiente y el performance organizacional, ocupando como proxy de esta última, en una primera instancia, la rentabilidad de las empresas (Rettab, Mellahi \& Ben, 2008; Shihping \& Chih-Lung, 2014; Simionescu \& Cristian, 2014; Wiengarten, Lo \& Lam, 2017; Susnaningsih \& Agung, 2018; Liu, Quan, Liu \& Bowen, 2019; Sitara, Rusmawati \& Norlida, 2019; Erhirhie \& 
Ekwueme, 2019; Siminica, Cristea, Sichigea, Georgiana \& Anghel, 2019). Otra parte de los trabajos realizados, buscan encontrar una relación significativa con variables relativas a los precios de las acciones puestas en los diferentes mercados internacionales de capital (Surroca, Tribó \& Waddock, 2010; Karagiorgos, 2010; Gregory, Tharyan \& Whittaker, 2014; Zhao, 2015; Cegarra, Reverte, Gómez \& Wensley, 2016).

Otros estudios, examinan la relación del consumo energético con la toma de decisiones de la gerencia, como es el caso, del trabajo de Aulí (2002), quien afirma que la inclusión de variables ambientales al proceso de toma de decisiones en la empresa, es ineludible y que la participación de la dirección debe reflejarse en la definición de objetivos ambientales. Investigaciones han asociado el consumo energético de las organizaciones con la intervención de los empleados de las compañías (Serna, 2010), estableciendo que para tener un resultado positivo en el ahorro y uso eficiente de la energía, es necesario implementar lo que denominó Administración Energética Empresarial (AEE), que toma en cuenta la participación activa de cada integrante de la compañía, desde los empleados hasta la alta gerencia, en el fomento de una cultura organizacional encaminada a la protección del medio.

Por último, la construcción de índices para medir el grado de sustentabilidad en las empresas, se ha convertido en una práctica recurrente, como lo demuestra el estudio realizado por Barrera et al. (2004), que desarrolla un índice para medir la sustentabilidad de las actividades industriales en la refinería Miguel Hidalgo o el trabajo de Sepúlveda et al. (2002), quienes construyen un índice de sostenibilidad (S3), el cual remite a un valor específico de desempeño de la unidad de análisis en un determinado período de tiempo. En Cuba, se construye un índice socioeconómico, que se utiliza para medir las actividades sustentables en empresas dedicadas al sector pecuario (Vázquez et al., 2011), el estudio de Carrillo (2014), a través de la aplicación de índices, mide la sustentabilidad de conjuntos urbanos en el Estado de México en el periodo 2002-2015. Sus resultados muestran que los conjuntos autorizados en Calimaya, durante el periodo 2002-2015, no son sustentables desde un análisis territorial. Umukoro et al. (2019) a través de la aplicación de un índice de sustentabilidad, analizan la relación existente entre el índice y la experiencia en el tema de cuidado al medio, por parte de los directivos de 10 organizaciones bancarias en Nigeria. Por último, el trabajo de Polo et al. (2019), muestra que las actividades sustentables realizadas por empresas industriales en el municipio de Tepeji del Rio, influyen significativamente en su esquema financiero, tal como lo describe el Índice de sustentabilidad y desempeño financiero. Es importante destacar, que los trabajos basados en índices, son aquellos que nos sirven de punto de partida para la construcción metodológica del presente estudio. Estas investigaciones, se encuentran directamente relacionadas con el presente trabajo. 


\section{Diseño y metodología}

En virtud de que el objetivo que se pretende en la presente investigación, es conocer si las practicas ahorradoras de energía eléctrica desarrolladas por empresas Hidalguenses, influyen en la estructura financiera de las mismas. En una primera instancia, se analiza el grado en que las empresas de la muestra, realizan actividades organizacionales respetuosas del medio ambiente, a través de la aplicación del instrumento de buenas prácticas para la reducción del consumo energético similar al propuesto en Observatorio industrial del sector textil y de la confección, (2011). En una segunda parte, se buscara conocer los factores intrínsecos y extrínsecos a la organización que influyen significativamente en el ahorro financiero.

\section{Determinación de variables Dependiente}

En virtud de que el instrumento de medición obtiene información respecto de aquellas compañías que realizan actividades sustentables y si estas representan un ahorro en sus gastos operativos, a través del método científico deductivo (Dávila, 2006), se genera el silogismo que indica, que el ahorro en gastos, genera un aumento en ingresos y por tanto, mayor utilidad. En tal caso, se desarrolla la siguiente ecuación, que permite obtener el Índice Financiero (IFIN), similar al calculado por Sepulveda et al., (2002); Barrera et al., (2004); Vázquez et al., (2011):

Donde:

$$
I F I N=\sum R(S i) / R(S i)+R(N o)
$$

IFIN = Índice Financiero

$\mathrm{R}(\mathrm{Si})=$ Respuestas cuyo valor es 1

$\mathrm{R}(\mathrm{No})=$ Respuestas cuyo valor es cero

\section{Independientes}

Para identificar las prácticas ahorradoras de energía que se desarrollan al interior de las empresas de la muestra, se construye un índice denominado "Índice de prácticas sustentables (IPS)", similar al utilizado por Céspedes (2011), Vázquez et al. (2011), Carrillo (2014), Polo et al. (2019), el IPS considera los resultados de 6 dimensiones (Servicio Contratado, Gestión y Ahorro Energético, Edificios, Instalaciones Industriales y Compromiso Organizacional). La expresión del índice es la siguiente:

Donde:

$$
\text { IPS }=\sum R s i+R n o / \sum M P
$$

IPS= Índice de Prácticas Sustentables

$\mathrm{Rsi}=$ Respuestas con valor de 1 o respuesta "si"

$\mathrm{Rno}=$ Respuestas con valor de 0 o respuesta "no"

MP= Máxima Puntuación del instrumento (seis dimensiones) 
La Tabla 1, muestra la definición de cada una de las variables independientes.

Tabla 1. Variables Independientes

\begin{tabular}{|c|c|c|}
\hline Nombre & Expresión & Definición \\
\hline Región & $\mathrm{RE}$ & $\begin{array}{l}\text { Variable dicotómica que toma el valor de } 1 \\
\text { cuando es de Tulancingo y } 2 \text { para Tepeji }\end{array}$ \\
\hline Edad del entrevistado & EDAD & $\begin{array}{l}\text { Variable de tipo categórico que toma el } \\
\text { valor de } 1 \text { cuando la edad se ubica de } 20 \text { a } \\
40 \text { años, } 2 \text { cuando la edad esta de } 41 \text { a } 60 \\
\text { años y } 3 \text { cuando es mayor de } 60 \text { años }\end{array}$ \\
\hline $\begin{array}{l}\text { Grado de escolaridad del } \\
\text { entrevistado }\end{array}$ & $\begin{array}{l}\text { ESCOLARIDA } \\
\text { D }\end{array}$ & $\begin{array}{l}\text { Variable de tipo categórico que toma el } \\
\text { valor de } 1 \text {, cuando el entrevistado presenta } \\
\text { estudios de secundaria, } 2 \text { bachillerato y } 3 \\
\text { para educación profesional }\end{array}$ \\
\hline $\begin{array}{l}\text { Experiencia en el extranjero del } \\
\text { entrevistado }\end{array}$ & EXP_EXT & $\begin{array}{l}\text { Variable de tipo dicotómico que toma el } \\
\text { valor de } 1 \text { para "si" y } 0 \text { para "no" }\end{array}$ \\
\hline $\begin{array}{l}\text { Experiencia en el sector del } \\
\text { entrevistado }\end{array}$ & EXP_SECTOR & $\begin{array}{l}\text { Variable categórica con tres valores, } 1 \\
\text { cuando se cuenta con experiencia de } 1 \text { a } 10 \\
\text { años, } 2 \text { de } 11 \text { a } 30 \text { años y } 3 \text { cuando la } \\
\text { experiencia del entrevistado es mayor de } \\
30 \text { años }\end{array}$ \\
\hline Índice de prácticas sustentables & IPS & $\begin{array}{l}\text { Índice de prácticas sustentables, calculado } \\
\text { como } \sum \mathrm{Rsi}+\mathrm{Rno} / \sum \mathrm{MPR} \text { por dimensión }\end{array}$ \\
\hline
\end{tabular}

Fuente: Elaboración propia

\section{Hipótesis}

Con el propósito de alcanzar los objetivos planteados en la presente investigación y considerando el valor del IFIN como variable dependiente, se establecen las siguientes hipótesis:

$\boldsymbol{H}_{1}$ : Las prácticas sustentables ahorradoras de energía eléctrica, mantienen influencia significativa con el IFIN

$\boldsymbol{H}_{2}$ : Las características intrínsecas y extrínsecas de las organizaciones, tales como (Región Geográfica de las empresas analizadas, el Grado de Escolaridad, la Experiencia en el Extranjero y la Experiencia en el Sector industrial por parte del entrevistado y el Número de Trabajadores), mantienen una relación significativa con el IFIN

\section{Muestra}

La muestra, está formada por 10 empresas industriales pertenecientes a dos municipios del Estado de Hidalgo (México), en específico de Tulancingo de Bravo y Tepeji del Río con cinco compañías por cada uno, el detalle se presenta en la Tabla 2. Las empresas numeradas con 9 y 10, pertenecientes al municipio de Tulancingo, solicitaron no dar a conocer su nombre. 
Tabla 2. Detalle de la Muestra

\begin{tabular}{|c|c|c|c|}
\hline Nombre & Sector & Región & $\mathrm{N}^{\circ}$ empleados \\
\hline KY2 kya2 & Textil Punto fino & Tulancingo & 60 \\
\hline Creaciones Regis & $\begin{array}{l}\text { Textil Punto } \\
\text { fino }\end{array}$ & Tulancingo & 25 \\
\hline Abraxas & $\begin{array}{l}\text { Textil Punto } \\
\text { fino }\end{array}$ & Tulancingo & 20 \\
\hline Empresa 9 & Textil acabados & Tulancingo & 30 \\
\hline Empresa 10 & Textil acabados & Tulancingo & 35 \\
\hline Flexisac S.A de C.V & Plásticos & Tepeji & 50 \\
\hline $\begin{array}{l}\text { Manufacturas Kaltex S.A de } \\
\text { C.V }\end{array}$ & Textil acabados & Tepeji & Más de 100 \\
\hline Distribuidora Textil Arttex S. & Textil confección & Tepeji & 370 \\
\hline Compañía Industrial de Tepeji & Textil acabados & Tepeji & 45 \\
\hline Puente sierra & Textil acabados & Tepeji & 45 \\
\hline
\end{tabular}

Fuente: Elaboración propia

\section{Análisis descriptivo}

El análisis del Índice de Practicas Sustentables (IPS), permite identificar que tan grande es el gap existente entre la realización de prácticas que reducen el consumo energético recomendadas en la literatura y aquellas que se desarrollan al interior de las organizaciones, esto se llevará a cabo a través de la aplicación del instrumento de buenas prácticas para la reducción del consumo energético similar al propuesto en Observatorio industrial del sector textil y de la confección, (2011). Los resultados del análisis descriptivo del IPS en función a la Región Geográfica, se presentan en la Tabla 3, donde podemos observar que las empresas pertenecientes al Municipio de Tepeji del Río, son quienes realizan el mayor número de actividades ahorradoras de energía con más del $60 \%$ de ellas, por el contrario, en las compañías de la región de Tulancingo, sus actividades de respeto al medio ambiente, no alcanzan el $50 \%$.

Tabla 3. Análisis descriptivo del comportamiento del IPS en función de la Región Geográfica

\begin{tabular}{l|llll}
\hline Región & Media & Mínimo & Máximo & Desv. típ. \\
\hline Tulancingo & 0.485 & 0.424 & 0.545 & 0.057 \\
Tepeji del Rio & 0.618 & 0.273 & 0.758 & 0.201 \\
\hline
\end{tabular}

Fuente: Elaboración propia

La Figura 1, muestra el comportamiento del IPS en función del grado de escolaridad del responsable de la empresa analizada, donde se puede observar que las compañías pertenecientes al municipio de Tepeji del Río y que son dirigidas por personas con un nivel educativo de secundaria, son quienes realizan actividades de mayor respeto al medio ambiente a través del ahorro de energía eléctrica con un valor superior a .75 
Figura 1. Comportamiento del IPS en función del nivel educativo del responsable organizacional

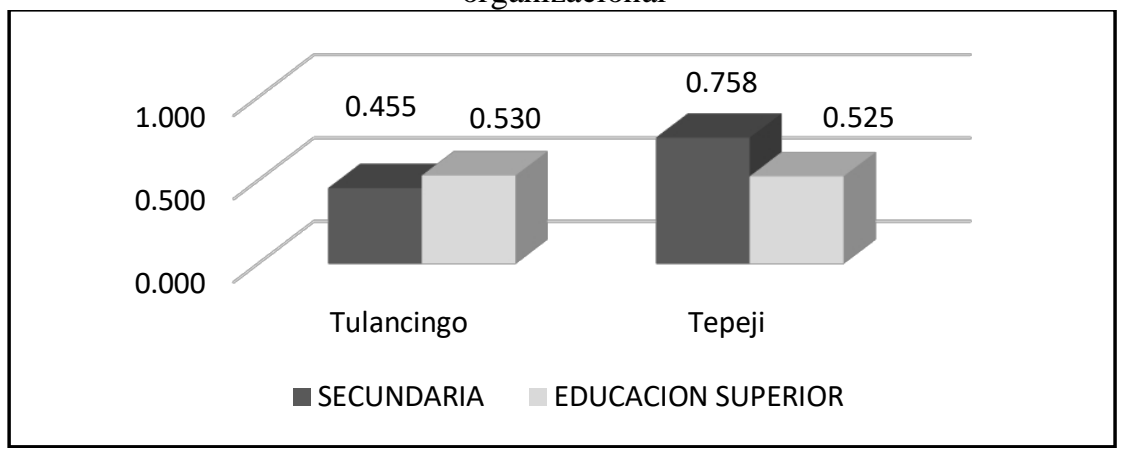

Fuente: Elaboración propia

Respecto a la experiencia en el extranjero que el responsable organizacional cuenta, el comportamiento del IPS presenta el mayor valor en las compañías pertenecientes al municipio de Tepeji del Río, cuyo responsable cuenta con experiencia en otro país, con más del 68\% de las prácticas sustentables recomendadas por el observatorio industrial. Por el contrario, el menor valor del índice lo presentan los responsables de las empresas de Tulancingo, a pesar de que cuentan con experiencia en el extranjero con un valor de apenas el $47 \%$ de actividades sustentables recomendadas (tabla 4).

Tabla 4. Comportamiento del IPS en función de la experiencia en el extranjero por parte del responsable organizacional

\begin{tabular}{c|cccccccccc}
\hline \multicolumn{4}{c}{ Municipio de Tulancingo } & \multicolumn{5}{c}{ Municipio de Tepeji del Río } \\
\hline $\begin{array}{c}\text { Experi } \\
\text { encia }\end{array}$ & Media & Mínimo & Máximo & $\begin{array}{c}\text { Desv. } \\
\text { típ. }\end{array}$ & $\begin{array}{c}\text { Experienci } \\
\text { a }\end{array}$ & Media & Mínim & Máxim & Desv. \\
NO & 0.545 & 0.545 & 0.545 &. & NO & 0.515 & 0.273 & 0.758 & 0.343 \\
& & & & & & & & & & \\
SI & 0.470 & 0.424 & 0.515 & 0.052 & SI & 0.687 & 0.636 & 0.758 & 0.063 \\
\hline
\end{tabular}

La tabla 5, presenta el análisis descriptivo del IPS en función de la experiencia que presenta el responsable organizacional en el sector económico de pertenencia, los resultados muestran de nueva cuenta a las empresas de Tepeji del Río, con la mayor cantidad de prácticas ahorradoras de energía eléctrica, tal como lo demuestra el valor del índice con más del 75\%. Por el contrario, el menor valor del IPS se encuentra en las compañías del municipio de Tulancingo con .477 puntos. 
Tabla 5. Comportamiento del IPS en función de la experiencia en el sector, por parte del responsable organizacional

\begin{tabular}{|c|c|c|c|c|c|c|c|c|c|}
\hline \multicolumn{5}{|c|}{ Municipio de Tepeji del Río } & \multicolumn{5}{|c|}{ Municipio de Tulancingo } \\
\hline Tiempo & $\begin{array}{c}\text { Medi } \\
\text { a }\end{array}$ & Mínimo & Máximo & $\begin{array}{l}\text { Desv. } \\
\text { típ. }\end{array}$ & Tiempo & Media & Mínimo & Máximo & $\begin{array}{c}\text { Desv. } \\
\text { típ. }\end{array}$ \\
\hline $\begin{array}{l}\text { De } 0 \text { a } 10 \\
\text { años }\end{array}$ & 0.455 & 0.273 & 0.636 & 0.257 & $\begin{array}{l}\text { De } 0 \text { a } 10 \\
\text { años }\end{array}$ & - & - & - & \\
\hline $\begin{array}{c}\text { De } 11 \text { a } 30 \\
\text { años }\end{array}$ & 0.712 & 0.667 & 0.758 & 0.064 & $\begin{array}{l}\text { De } 11 \text { a } \\
30 \text { años }\end{array}$ & 0.477 & 0.424 & 0.545 & 0.062 \\
\hline $\begin{array}{l}\text { De } 31 \text { en } \\
\text { adelante }\end{array}$ & 0.758 & 0.758 & 0.758 & . & $\begin{array}{l}\text { De } 31 \text { en } \\
\text { adelante }\end{array}$ & 0.515 & 0.515 & 0.515 & 0.000 \\
\hline
\end{tabular}

\section{Análisis multivariante (Técnicas de análisis)}

Con el propósito de contrastar las hipótesis planteadas y conociendo que la distribución de los datos es normal (Test de Shapiro W), se procede a la utilización de pruebas paramétricas o multivariantes (modelos con mayor potencia, que sus homólogos no paramétricos, Gómez y Soria, 2001), específicamente a través de modelos de regresión lineal. Para evitar problemas de heterocedasticidad se aplica el test de White con el programa Stata. El factor inflacionario de la varianza (FIV), permite señalar el nivel de multicolinealidad en las variables del modelo, donde a mayor valor del FIV, mayor problema o colinealidad tiene la variable, como regla práctica se tiene que si el FIV de una variable, es superior a 10, se dice que la misma presenta multicolinealidad (Guajarati \& Porter, 2010), por ello, en nuestro caso, los valores del VIF del Statistical Package for the Social Sciences (SPSS) versión 15 , de las variables, oscilan de 1.407 a 2.656 , por lo que, se puede sostener que el modelo de regresión lineal, no presenta problemas de multicolinealidad. El modelo para el Índice Financiero, es el siguiente:

\section{$I F I N=\beta_{0}+\beta_{1} R G+\beta_{2} E S C+\beta_{3} E X P_{-} E X T+\beta_{4} E X P \_S E C+\beta 5 I P S+\varepsilon$}

Donde:

IFIN: Índice de financiero

RG: $\quad$ Región Geográfica

ESC: $\quad$ Grado de escolaridad del encuestado

EXP_EXT: Experiencia en el extranjero del encuestado

EXP_SEC: Experiencia en el sector del encuestado

IPS: Î́ndice de prácticas sustentables

\section{Resultados}

Los resultados de la aplicación del modelo de regresión lineal se recogen en la Tabla 6, donde se observa que existe una relación estadísticamente significativa de tipo positivo, entre el índice financiero (IFIN) y las variables Escolaridad del encuestado ( $p$-value de 0.100 al $90 \%$ de confianza), Número de trabajadores ( $p$-value de 0.100 al $90 \%$ de confianza) y 
el Índice de prácticas sustentables (IPS) (p-value de 0.014 al 95\% de confianza), lo que significa en el primer caso, que aquellos encargados de las organizaciones que presentan mayor grado académico, son los que propician mayor ahorro en sus gastos de operación y por tanto, aumentan el índice financiero, como lo indica el signo de su coeficiente. Respecto al número de trabajadores, las compañías analizadas con plantillas grandes, son empresas que aumentan el IFIN y por tanto, aumentan sus utilidades, por último, se podría afirmar que la adopción de prácticas sustentables (ahorradoras de energía eléctrica) por parte de las compañías analizadas, genera un ahorro en los gastos de operación y por tanto, la obtención de mayores utilidades, de esta forma se aceptan la $\boldsymbol{H}_{1}$ : Las prácticas sustentables ahorradoras de energía eléctrica, mantienen influencia significativa con el IFIN. No son significativas las variables Región Geográfica, Experiencia en el Extranjero y Experiencia en el Sector del entrevistado. Se acepta parcialmente la $\boldsymbol{H}_{2}$ : Las características intrínsecas y extrínsecas de las organizaciones, tales como (Región Geográfica de las empresas analizadas, el Grado de Escolaridad, la Experiencia en el Extranjero y la Experiencia en el Sector industrial por parte del entrevistado y el Número de Trabajadores), mantienen una relación significativa con el IFIN, por los casos de la Escolaridad y el número de trabajadores.

Tabla 6. Modelo de regresión del Índice Financiero (IFIN)

\begin{tabular}{|c|c|c|c|c|}
\hline \multicolumn{5}{|c|}{$I F I N=\beta 0+\beta 1 R E+\beta 2 E D+\beta 3 E S C+\beta 4 E X P \_E X T+\beta 5 E X P \_S E C+\beta 11 I P S+\varepsilon$} \\
\hline Variable & Coeficiente & Error Tip & Sig. & VIF \\
\hline Constante & -0.202 & 0.114 & 0.176 & \\
\hline Región & 0.035 & 0.044 & 0.484 & 2.167 \\
\hline $\begin{array}{l}\text { Grado de escolaridad del } \\
\text { entrevistado }\end{array}$ & 0.057 & 0.025 & 0.100 & 2.127 \\
\hline $\begin{array}{l}\text { Experiencia en el extranjero del } \\
\text { entrevistado }\end{array}$ & -0.055 & 0.039 & 0.252 & 1.407 \\
\hline $\begin{array}{l}\text { Experiencia en el sector del } \\
\text { entrevistado }\end{array}$ & 0.029 & 0.038 & 0.503 & 2.656 \\
\hline Número de Trabajadores & $2.73 \mathrm{E}-006$ & 0.000 & 0.100 & 1.432 \\
\hline IPS & 0.795 & 0.154 & 0.014 & 2.328 \\
\hline R-cuadrada & \multicolumn{4}{|c|}{0.977} \\
\hline ANOVA & \multicolumn{4}{|c|}{0.015} \\
\hline
\end{tabular}

\section{Conclusiones}

El propósito de la presente investigación, recae en identificar si las prácticas de ahorro de energía realizadas en empresas industriales del Estado de Hidalgo, ajustadas de manera similar al modelo propuesto en Observatorio industrial del sector textil y de la confección, (2011), mantienen una relación significativa con el IFIN y si esta, se traduce como un ahorro en términos financieros aumentado por derivación lógica las utilidades de las 
organizaciones de la muestra. Los resultados de los análisis descriptivos del comportamiento del IPS por región, nos podrían permitir afirmar que las compañías ubicadas en el municipio de Tepeji del Río, son las que mayor compromiso presentan con el medio ambiente, sin embargo, sus actividades apenas superan el $50 \%$ de las recomendadas por el observatorio industrial.

Los resultados del análisis multivariante, nos permiten observar que la adopción de prácticas sustentables (ahorradoras de energía eléctrica), por parte de las compañías analizadas, genera un ahorro en los gastos de operación y, por tanto, la obtención de mayores utilidades, lo que se traduce en un beneficio no solo ambiental, sino también social, en virtud de que aquellas empresas con mayores niveles de rentabilidad, mayores serán las aportaciones al fisco, vía pago de contribuciones.

En definitiva, aunque el tema de sustentabilidad se ha mencionado desde unos años atrás, aún nos falta mucho por aprender de aquellos países donde el medio ambiente es en verdad tomado en serio, se recomienda establecer códigos de mejores prácticas de ahorro energético para este tipo de empresas y cuyo cumplimiento sea reglamentado y observado por los organismos encargados de la vigilancia al medio ambiente, pues se ha demostrado que el compromiso organizacional aumenta el número de prácticas de ahorro energético, impactando significativamente las estructuras financieras en la determinación de utilidades.

\section{References:}

1. Agüero, F. (2002). La Responsabilidad Social Empresarial en América Latina: Argentina, Brasil, Chile, Colombia, México y Perú”, Escuela de Estudios Internacionales, Universidad de Miami.

2. Arreguín, G., Álvarez, G. \& Sánchez, D. (2019). La teoría institucional y la responsabilidad social en el sector hotelero en la ciudad de Celaya, Guanajuato. Tourism and Hospitality International Journal, 12(1), 5069.

3. Anzures, T., Briones, V. \& Galarza, D. (2017). “Análisis de sustentabilidad de las pymes de confecciones con potencial exportador de la zona 8 del Ecuador", $2^{\circ}$ Congreso Internacional en Administración de Negocios Internacionales, Medellín, Colombia.

4. Aulí, E. (2002). "Integración de los factores ambientales a las estrategias empresariales", ICE Desarrollo Sostenible, no. 800, p.p. 139-148.

5. Barrera, A., Saldivar, A., Nava, M., Ortíz, S., Aguilar, S. \& Villaseñor, E. (2004). "Índice de sustentabilidad industrial: Refinería Miguel Hidalgo", Problemas del Desarrollo, Vol- 35, Nº 137, pp 77-93. 
6. Begoña, M. (2010). "Ahorro y gestión eficiente de la energía", Instituto Sindical de Trabajo, Ambiente y Salud (ISTAS), Paralelo edición, S.A.

7. Briseño, A., Lavín, J. \& García, F. (2011). “Análisis exploratorio de la responsabilidad social empresarial y su dicotomía en las actividades sociales y ambientales de la empresa", Contaduría y Administración, No. 233, pp 73-83.

8. Carrillo, A., Jiménez, P. \& Carreño, F. (2014). "Sistema de indicadores territoriales para evaluar la sustentabilidad de conjuntos urbanos en el estado de México: caso de estudio Calimaya", $21^{\circ}$ Encuentro Nacional sobre Desarrollo Regional en México. Mérida, Yucatán del 15 al 18 de noviembre de 2016. AMECIDER - ITM

9. Cegarra, J., Reverte, C., Gómez, E. \& Wensley, A. (2016). “Linking social and economic responsibilities with financial performance: The role of innovation", European Management Journal, Vol. XXX, pp. 110.

10. Centro del Cambio Global y la Sustentabilidad, A.C (2020). CCGS (Extraído de http://ccgss.org/sustentabilidad/)

11. Cespedes (2001). "Índice de sustentabilidad ambiental: sustentabilidad ambiental comparada en las entidades federativas de México. Centro de estudios del sector privado para el desarrollo sustentable. $220 \mathrm{p}$

12. Conferencia de las Naciones Unidas Sobre el Desarrollo Sostenible (Río+20, 2012). Disponible en:

http://www.un.org/es/sustainablefuture/about.shtml (Fecha de consulta: 10 de diciembre de 2019).

13. Dávila, G. (2006). "El razonamiento inductivo y deductivo dentro del proceso investigativo en ciencias experimentales y sociales", Revista de Educación, Año 12, Número Extraordinario, pp. 180-205

14. Decenio Internacional para la Acción "el agua Fuente de Vida" (2005 - 2015). Disponible en:

http://www.un.org/spanish/waterforlifedecade/water_and_energy.sht $\mathrm{ml}$ (Fecha de consulta: 10 de diciembre de 2019).

15. De la Cuesta, M. \& Valor, C. (2003). "Responsabilidad social de la empresa. Concepto, medición y desarrollo en España", Boletín Económico de ICE, No 2755, pp. 7-20.

16. Erhirhie, E. \& Ekwueme, M. (2019). “Corporate Social Sustainability Reporting and Financial Performance of Oil and Gas Industry in Nigeria", International Journal of Accounting, Finance and Risk Management, 4(2), pp. 44-60.

17. Gregory, A., Tharyan, R. \& Whittaker, J. (2014). "Corporate Social Responsibility and Firm Value: Disaggregating the Effects on Cash Flow, Risk and Growth", Ethics, (124), pp. 633-657. 
18. Gómez \& Soria. (2001). Pruebas de significación en Bioestadística. Revista de diagnóstico Biológico, 50(4). Recuperado de http://scielo.isciii.es/scielo.php?script=sci_arttext\&pid=S003479732001000400008

19. Guajarati, D. \& Porter, D. (2010). Flexibilización de los supuestos del modelo clásico. En Econometría. (pp. 327-329). México, D.F: MC Graw Hill

20. Informe de las Naciones Unidas Sobre el Desarrollo de los Recursos Hídricos en el Mundo (2014). Organización de las Naciones Unidas para la Educación, la Ciencia y la Cultura.

21. Karagiorgos, T. (2010). "Corporate Social Responsibility and Financial Performance: An Empirical Analysis on Greek Companies", European Research Studies, Volume XIII, Issue (4), pp. 85-108.

22. Liu, N., Quan, G., Liu, C. \& Bowen. D. (2019). "Corporate Social Responsibility and Financial Performance: A Quantile Regression Approach", Sustainability, 11 (13), pp 1-22.

23. Observatorio Industrial del Sector Textil y de la Confección (2011). Disponible en:

http://www.minetur.gob.es/industria/observatorios/SectorTextil/Activ idades/2011/CIE,\%20FITAG-UGT,\%20FITEQA-

CCOO/Necesidades_Formacion_Parte_I.pdf, (Fecha de consulta: 10 de junio de 2019).

24. Polo, S., Villegas, E., Duana, D. \& Benítez, D. (2019). "El uso de prácticas sustentables y su influencia en la información financiera de empresas industriales del municipio de Tepeji del Rio, Hidalgo", Actualidad Contable FACES, Año 21, № 38, pp. 126-137.

25. Rettab, B., Mellahi, K. \& Ben, A. (2008). "A Study of Management Perceptions of the Impact of Corporate Social Responsibility on Organisational Performance in Emerging Economies: The Case of Dubai", Journal of Business Ethics, 89(3), pp 371-390.

26. Sepúlveda, S., Chavarría, H., Castro, A., Rojas, P., Picado, E. \& Bolaños, D. (2002). "Metodología para estimar el nivel de desarrollo sostenible en espacios territoriales". IICA.

https://ecaths1.s3.amazonaws.com/desarrollosostenible/176848813.M etodologia\%20e. (Fecha de búsqueda diciembre 2019).

27. Serna, C. (2010). "Gestión energética empresarial una metodología para la reducción de consumo de energía" Producción + Limpia, vol. 5, no. 2, p.p. 107-126.

28. Shihping, K. \& Chih-Lung, Y. (2014). "Corporate social performance: why it matters? Case of Taiwan", Chinese Management Studies, Vol. 8 No. 4, pp 704-716. 
29. Siminica, M., Cristea, M., Sichigea, M., Georgiana, G. \& Anghel, I. (2019). "Well-Governed Sustainability and Financial Performance: A New Integrative Approach", Sustainability, (11), pp. 1-21.

30. Simionescu, L. \& Cristian, G. (2014). "Corporate social responsibility and corporate performance: empirical evidence from a panel of the Bucharest Stock Exchange listed companies" M \& M, Vol. 9, No. 4, pp. 439-458.

31. Sitara, K., Rusmawati, B. \& Norlida, A. (2019). "Legitimizing the role of corporate boards and corporate social responsibility in the performance of listed companies in Malaysia", Indian Journal of Corporate Governance, vol. 12 (2), pp. 125-141.

32. Surroca, J., Tribó, J. \& Waddock, S. (2010). "Corporate responsibility and financial performance: the role of intangible resources", Strategic Management Journal, (31), pp. 463-490.

33. Susnaningsih, M. \& Agung, P. (2018). "Disclosure of Corporate Social Responsibility and Financial Performance: A Case Study of StateOwned Companies", Sosial Budaya, Vol. 15, No. 1, pp 11-18.

34. Umukoro, O., Uwuigbe, O., Uwuigbe, U., Adegboye, A., Ajetunmobi, O. \& Nwaze, C. (2019). "Board Expertise and Sustainability Reporting in Listed Banks in Nigeria", Earth and Environmental Science, $N^{\circ} 331$.

35. Vázquez, Y., Guerra, C. \& Sánchez, O. (2011). "Modelación Estadístico-Matemática para el estudio de la sostenibilidad socioeconómica en el sector agrícola-pecuario del municipio San José de las Lajas, provincia Mayabeque", Ciencias Técnicas Agropecuarias, Vol. 20, No4, pp 69-74.

36. Wiengarten, F., Lo, CH. \& Lam, J. (2017). "How does Sustainability Leadership Affect Firm Performance? The Choices Associated with Appointing a Chief Officer of Corporate Social Responsibility", Journal of Business Ethics, 140 (3), pp 477-493.

37. Zhao, R. (2015). "Sustainability and firm valuation: An international investigation", International Journal of Accounting and Information Management, 23(3). 